VOL. 49 (1994) [111-116]

\title{
A NOTE ON FRÉCHET AND APPROXIMATE SUBDIFFERENTIALS OF COMPOSITE FUNCTIONS
}

\author{
A. Jourani and L. Thibault
}

The aim of this note is to present in the reflexive Banach space setting a natural and simple proof of the formula of the approximate subdifferential of a composite function.

\section{INTRODUCTION}

In [7] we have shown how a subdifferential formula of the form

$$
\partial(g \circ G)\left(x_{0}\right) \subset \bigcup_{y^{*} \in \theta_{g}\left(G\left(x_{0}\right)\right)} \partial\left(y^{*} \circ G\right)\left(x_{0}\right)
$$

allows the establishment in a natural way of the existence of Kuhn-Tucker multipliers for general mathematical programming problems whenever the metric regularity (qualification of the constraints) is satisfied. Unfortunately such a formula does not hold in general. The corresponding formula for Clarke's subdifferential (see [1]) requires taking the (closed) convex closure of the second member of (1) and hence the simple method in [7] may not been used to show the existence of multipliers. This formula (1) has been proved by Ioffe in [5] for locally Lipschitz functions $g$ and mappings $G$ admitting compact prederivatives and by ourselves in [8] for the more general class of strongly compactly Lipschitzian mappings $G$. Both proofs in [5] and [8] are very technical. The aim of this note is to present, in the special case of reflexive Banach spaces, a much less technical proof. Using the Fréchet subdifferentials in the elegant way of Kruger and Mordukhovich [10] (after the pioneering work by Mordukhovich [11]) is the key to our approach in this note.

\section{SUBDIFFERENTIAL FORMULAE FOR COMPOSITE FUNCTIONS}

Let $X$ and $Y$ be two Banach spaces, $B_{X}$ and $B_{Y}$ their closed unit balls and $X^{*}$ and $Y^{*}$ their topological duals. Let also $f$ be a function from $X$ into $\mathbb{R} \cup\{+\infty\}$. For any $\varepsilon>0$ one defines the Fréchet $\varepsilon$-subdifferential $\delta_{F}^{e} f(x)$ of $f$ at a point $x$ where $f$

Received 22nd February, 1993

Copyright Clearance Centre, Inc. Serial-fee code: 0004-9729/94 \$A2.00+0.00. 
is finite by $x^{*} \in \delta_{F}^{e} f(x)$ if and only if there is a neighbourhood $X_{0}$ of $x$ in $X$ such that

$$
\left\langle x^{*}, u-x\right\rangle \leqslant f(u)-f(x)+\varepsilon\|u-x\|
$$

for all $u \in X_{0}$.

The limiting Fréchet subdifferential of $f$ at a point $x_{0}$ (with $f\left(x_{0}\right)<+\infty$ ) is (see Kruger [9])

$\partial_{F} f\left(x_{0}\right)=\left\{x^{*} \in X^{*}: x^{*}=w^{*}-\lim _{n \rightarrow \infty} x_{n}^{*}, x_{n}^{*} \in \delta_{F}^{e_{n}} f\left(x_{n}\right), x_{n} \underset{n \rightarrow+\infty}{\stackrel{f}{\rightarrow}} x_{0}\right.$ and $\left.\varepsilon_{n} \downarrow 0\right\}$, where $w^{*}$ denotes the weak-star topology and $x_{n} \underset{n \rightarrow+\infty}{\stackrel{f}{\rightarrow}} x_{0}$ means that $x_{n} \rightarrow x_{0}$ and $f\left(x_{n}\right) \rightarrow f\left(x_{0}\right)$.

This subdifferential is always included in that of Clarke and if $\Psi(e p i f ;$.$) denotes$ the indicator function of the epigraph of $f$, then (see [9])

$$
\partial_{F} f\left(x_{0}\right)=\left\{x^{*} \in X^{*}:\left(x^{*},-1\right) \in \partial_{F} \Psi\left(\text { epif; } x_{0}, f\left(x_{0}\right)\right)\right\}
$$

If $X$ is reflexive, then, whenever $f$ is Lipschitz at $x_{0}$, (see [6]) $\partial_{F} f\left(x_{0}\right)$ is equal to the approximate subdifferential $\partial_{A} f\left(x_{0}\right)$ and $\partial_{F} f$ is $w^{*}$-sequentially upper semicontinuous at $x_{0}$, that is for $x_{n} \rightarrow x_{0}, x_{n}^{*} \in \partial_{F} f\left(x_{n}\right)$ and $x_{n}^{*} \stackrel{w^{*}}{\rightarrow} x^{*}$ one has $x^{*} \in \partial_{F} f\left(x_{0}\right)$.

The following result has been proved by Kruger [9] in a more general setting.

ThEOREM 1. [9] Let $f: X \rightarrow \mathbb{R} \cup\{+\infty\}$ be finite at $x_{0}$ and lower semicontinuous near $x_{0}$ and let $g: X \rightarrow \mathbb{R}$ be locally Lipschitz around $x_{0}$. If $X$ has an equivalent Fréchet differentiable norm at all nonzero points, then

$$
\partial_{F}(f+g)\left(x_{0}\right) \subset \partial_{F} f\left(x_{0}\right)+\partial_{F} g\left(x_{0}\right)
$$

Before establishing our main result we need to recall the notion of compactly Lipschitzian mappings introduced by the second author in [12].

A mapping $G: X \rightarrow Y$ is compactly Lipschitzian at a point $x_{0}$ if there exist a multivalued function $K: X \rightrightarrows C o m p(Y)$, where $\operatorname{Comp}(Y)$ denotes the set of all norm compact subsets of $Y$, a function $r:(0,1] \times X \times X \rightarrow \mathbb{R}_{+}$and neighbourhoods $X_{0}$ of $x_{0}$ and $V$ of zero in $X$ satisfying the following properties

(i) $\lim _{\substack{x \rightarrow z_{0} \\ t \downarrow 0}} r(t, x, v)=0$ for any $v \in X$ and $\lim _{\substack{(x, v) \rightarrow\left(x_{0}, 0\right) \\ t \downarrow 0}} r(t, x, v)=0$;

(ii) for all $x \in X_{0}, v \in V$ and $t \in(0,1]$

$$
t^{-1}(G(x+t v)-G(x)) \in K(v)+r(t, x, v) B_{Y}
$$

(iii) $K(0)=\{0\}$ and $K$ is upper semicontinuous at zero. 
Every compactly Lipschitzian mapping is locally Lipschitz and in finite dimensional spaces both concepts coincide. In [12] several examples are given.

The following result is a direct consequence of Lemma 2.5 in $\mathrm{El} \mathrm{Abdouni} \mathrm{and}$ Thibault [3] and of its proof. See also proposition 2.3 in Jourani and Thibault [8] and Theorem 1.5 in Glover and Craven [4].

LEMMA 2. Let $G: X \rightarrow Y$ be a mapping which is compactly Lipschitzian at $x_{0}$. If $\left(y_{n}^{*}\right) w^{*}$-converges to zero in $Y^{*}$, if $\left(x_{n}\right)\|\|$-converges to $x_{0}$ in $X$ and if $x_{n}^{*} \in \partial_{C}\left(y_{n}^{*} \circ G\right)\left(x_{n}\right)$ then $\left(x_{n}^{*}\right)_{n} w^{*}$-converges to zero in $X^{*}$. (Here $\partial_{C}$ denotes the Clarke subdifferential (see [1]).)

We can now establish our main result.

THEOREM 3. Let $G: X \rightarrow Y$ be a mapping which is compactly Lipschitzian at $x_{0} \in X$ and let $g: Y \rightarrow \mathbb{R}$ be a function which is Lipschitz around $y_{0}:=G\left(x_{0}\right)$. Assume that $X$ is reflexive and $Y$ admits an equivalent norm which is Fréchet differentiable at all nonzero points. Then

$$
\partial_{F}(g \circ G)\left(x_{0}\right) \subset \bigcup_{y^{*} \in \theta_{F} g\left(G\left(x_{0}\right)\right)} \partial_{F}\left(y^{*} \circ G\right)\left(x_{0}\right) .
$$

Proof: Fix $x^{*} \in \partial_{F}(g \circ G)\left(x_{0}\right)$. If we denote by $\Psi(g r G ;$.$) the indicator function$ of the graph of $G$ we see that

$$
g \circ G(x) \leqslant s(x, y)
$$

for all $(x, y) \in X \times Y$, where $s(x, y)=g(y)+\Psi(g r G ; x, y)$. So for any $\varepsilon>0$ and $u^{*} \in \delta_{F}^{e}(g \circ G)(x)$ we have $\left(u^{*}, 0\right) \in \delta_{F}^{e} s(x, G(x))$. Therefore by Theorem 1 (note that $X$ and so $X \times Y$ admit equivalent Fréchet differentiable norms (see [2])) we show

$$
\partial_{F}(g \circ G)\left(x_{0}\right) \times\{0\} \subset \partial_{F} s\left(x_{0}, y_{0}\right) \subset\{0\} \times \partial_{F} g\left(y_{0}\right)+\partial_{F} \Psi\left(g r G ; x_{0}, y_{0}\right)
$$

and hence there exists $y^{*} \in \partial_{F} g\left(y_{0}\right)$ such that $\left(x^{*},-y^{*}\right) \in \partial_{F} \Psi\left(g r G ; x_{0}, y_{0}\right)$.

Choose a sequence $\left(x_{n}^{*},-y_{n}^{*}\right) \stackrel{w^{*}}{\rightarrow}\left(x^{*},-y^{*}\right)$ with $\left(x_{n}^{*},-y_{n}^{*}\right) \in \delta_{F}^{\varepsilon_{n}} \Psi\left(g r G ; x_{n}, G\left(x_{n}\right)\right)$, $x_{n} \rightarrow x_{0}$ and $\varepsilon_{n} \downarrow 0$. If $k_{G}$ denotes a Lipschitz constant of $G$ at $x_{0}$ and if we set $\theta_{n}=\varepsilon_{n}\left(1+k_{G}\right)$, then we have for $x$ near $x_{n}$

$$
\begin{aligned}
\left\langle x_{n}^{*}, x-x_{n}\right\rangle-\left\langle y^{*}, G(x)-G\left(x_{n}\right)\right\rangle & \leqslant \varepsilon_{n}\left(\left\|x-x_{n}\right\|+\left\|G(x)-G\left(x_{n}\right)\right\|\right) \\
& \leqslant \theta_{n}\left\|x-x_{n}\right\|
\end{aligned}
$$

and hence

$$
\begin{aligned}
x_{n}^{*} & \in \partial_{F}\left(y_{n}^{*} \circ G+\theta_{n}\left\|\cdot-x_{n}\right\|\right)\left(x_{n}\right) \\
& \subset \partial_{F}\left(y^{*} \circ G\right)\left(x_{n}\right)+\partial_{F}\left(\left(y_{n}^{*}-y^{*}\right) \circ G\right)\left(x_{n}\right)+\theta_{n} B_{X^{*}}
\end{aligned}
$$


So $x_{n}^{*}=u_{n}^{*}+v_{n}^{*}+\theta_{n} b_{n}^{*}$ with $u_{n}^{*} \in \partial_{F}\left(y^{*} \circ G\right)\left(x_{n}\right), b_{n}^{*} \in B_{X}{ }^{*}$ and $v_{n}^{*} \in \partial_{F}\left(\left(y_{n}^{*}-y^{*}\right) \circ G\right)$ $\left(x_{n}\right) \subset \partial_{C}\left(\left(y_{n}^{*}-y^{*}\right) \circ G\right)\left(x_{n}\right)$. By Lemma 2 we have $v_{n}^{*} \stackrel{w^{*}}{\rightarrow} 0$ and hence $u_{n}^{*} \stackrel{w^{*}}{\rightarrow} x^{*}$. Thus by the $w^{*}$-sequential upper semicontinuity of $\partial_{F}\left(y^{*} \circ G\right)$ at $x_{0}$ we conclude that $x^{*} \in \partial_{F}\left(y^{*} \circ G\right)\left(x_{0}\right)$.

Denoting by $\partial_{A}$ the approximate subdifferential (see [5]), we see that, in the reflexive Banach space setting, the above approach gives a direct proof of the approximate subdifferential formula of composite functions. This proof is much less technical than the ones given by Ioffe in [5] for mappings with compact prederivatives and ourselves in [8] for strongly compactly Lipschitzian mappings. But we have to say that this approach does not work for general Banach spaces.

COROLlaRY 4. Let $G: X \rightarrow Y$ be a mapping which is compactly Lipschitzian at $x_{0} \in X$ and let $g: Y \rightarrow \mathbb{R}$ be a function which is Lipschitz around $y_{0}:=G\left(x_{0}\right)$. If $X$ and $Y$ are reflexive, then

$$
\partial_{A}(g \circ G)\left(x_{0}\right) \subset \bigcup_{y^{*} \in \theta_{A} g\left(G\left(x_{0}\right)\right)} \partial_{A}\left(y^{*} \circ G\right)\left(x_{0}\right)
$$

Proof: It is known (see [2]) that $Y$ admits an equivalent norm which is Fréchet differentiable at all nonzero points. As the approximate and Fréchet limiting subdifferentials of Lipschitz functions coincide for reflexive Banach spaces (this follows from the proof of Lemma 4 in [6]), the result is a direct consequence of the above theorem.

REMARK. Note that (in the reflexive Banach setting ) we only require compact Lipschitzness of $G$ instead of strongly compact Lipschitzness.

\section{REFERENCES}

[1] F.H. Clarke, Optimization and nonsmooth analysis (Wiley-Interscience, New York, 1983).

[2] J. Diestel, Geometry of Banach spaces-selected topics, Lecture Notes in Mathematics 485 (Springer Verlag, New York, 1975).

[3] B. El Abdouni and L. Thibault, 'Lagrange multipliers for Pareto nonsmooth programming problems in Banach spaces', Optimization 26 (1992), 277-285.

[4] B.M. Glover and B.D. Craven, 'A Fritz John optimality condition using the approximate subdifferential', Preprint (1992).

[5] A.D. Ioffe, 'Approximate subdifferentials and applications 3 : Metric theory', Mathematika 36 (1989), 1-38.

[6] A.D. Ioffe, 'Proximal analysis and aproximate subdifferentials', J. London Math. Soc. 41 (1990), 175-192.

[7] A. Jourani and L. Thibault, 'Approximations and metric regularity in mathematical programming in Banach space', Math. Oper. Res. 18 (1993), 390-401. 
[8] A. Jourani and L. Thibault, 'The approximate subdifferentials of composite functions', Bull. Austral. Math. Soc. 47 (1993), 443-455.

[9] A.Ya. Kruger, 'Properties of generalized differentials', Siberian Math. J. 26 (1985), 54-66.

[10] A.Ya. Kruger and B.S. Mordukhovich, 'Extreme points and the Euler equations in nondifferentiable optimization problems', Dokl. Akad. Nauk. BSSR 24 (1980), 684-687.

[11] B.S. Mordukhovich, 'Maximum principle in the optimal time control problem with nonsmooth constraints', J. Appl. Math. Mech. 40 (1976), 960-969.

[12] L. Thibault, 'Subdifferential of compectly lipschitzian vector-valued functions', Travaux du séminaire d'analyse convexe 8 (1978), Fascicule I. Montpellier.

Université de Bourgogne

Laboratoire d'Analyse Numérique

B.P. 138-21004 - Dijon Cedex

France
Université Montpellier II

Dept. des Sciences Mathématiques

34095 - Montpellier Cedex 5

France 